\title{
Decreasing readmissions: it can be done but one size does not fit all
}

\section{Finlay A McAlister}

Correspondence to Dr Finlay A McAlister, Division of General Internal Medicine and Patient Health Outcomes Research and Clinical Effectiveness Unit, University of Alberta, 2F1.21 WMC, University of Alberta Hospital, 8440112 Street, Edmonton, Alberta, Canada T6G 2R7; Finlay.McAlister@ualberta.ca

Received 13 August 2013 Accepted 13 August 2013 Published Online First 4 September 2013

\section{SLinked}

- http://dx.doi.org/10.1136/ bmjqs-2013-001901

To cite: McAlister FA. BMJ Qual Saf 2013;22:975-976.
Readmissions within 30 days of discharge are common, costly and hazardous-as such, efforts to reduce readmissions are a major focus in virtually all healthcare systems. ${ }^{1}$ In particular, policy makers have focused on heart failure (HF) as it is one of the most common reasons for hospitalisation in the developed world, HF patients already have the highest 30 -day readmission rates and despite substantial attention over the past decade to this problem the risk of post-discharge adverse events is actually increasing among patients with HF. ${ }^{1}{ }^{2}$ While a number of interventions have been suggested to reduce readmission rates, their effectiveness in clinical practice has often been disappointing when rigorously evaluated. ${ }^{3}$

However, in this issue of BMJ Quality of Safety, Amarasingham and colleagues report a remarkably successful programme associated with a $27 \%$ relative reduction (a 5\% absolute reduction) in 30 -day all-cause readmission rates in HF patients at Parkland Hospital in Dallas. ${ }^{4}$ This effect size was achieved even though the programme was only offered to approximately a quarter of discharged patients, was only deployed on weekdays (weekend discharges actually exhibit the highest rate of readmissions) $)^{5}$ and despite the fact that only a minority of readmissions may be truly preventable. ${ }^{6}{ }^{7}$ Indeed, most readmissions may be attributable to progression of disease or contextual factors such as socioeconomic status, the availability of home support and outpatient follow-up resources rather than poor quality of inpatient care. ${ }^{8-10}$ The size of the intervention effect reported by Amarasingham is all the more remarkable since it was achieved in urban indigent patients-the population thought to be most difficult in which to achieve improvements in post-discharge outcomes.

Although the Amarasingham study was a controlled before-after study rather than a randomised clinical trial, it does provide reasonably strong evidence for a true effect from their discharge intervention given the magnitude of the effect size, the dose-response relationship outlined in their Table 3 (readmission rates were even lower among those patients receiving more elements of the discharge intervention than those receiving less elements) and the lack of change in readmission rates over the same time frame for their controls. Of note, they reported data for three different sets of controls from their institution, including patients with (i) pneumonia, (ii) acute myocardial infarction and (iii) 'next most deserving' HF (ie, those HF patients with readmission risk scores just below those who qualified for their intervention). Another control group could have been contemporaneous HF patients admitted to similar hospitals in the Dallas area without any chance of exposure to the discharge programme. In the absence of such data, we can at least be reassured that other studies have reported minimal secular trends for 30 -day readmission rates after $\mathrm{HF}$ hospitalisations in the study years. ${ }^{2} 11$

The study does, however, raise two unanswered questions. First, we are not provided any information on the impact of their intervention on outpatient resources. Many discharge interventions increase outpatient or emergency department visits, ${ }^{3}$ and we need such information to determine if their intervention delivers good value for money from a broader perspective. Second, the investigators focused on patients being discharged back to the community. However, a substantial proportion of HF patients (up to $20 \%$ in the USA) are discharged to skilled nursing facilities and, even after adjusting for demographics and comorbidity profiles, these patients exhibit the highest risk of 30 -day readmissions. ${ }^{12}$ There is clearly a need for increased research attention to the transitions between acute care and long-term care facilities. 
Why was this intervention so successful while others have failed? We cannot judge the impact of individual elements of their discharge transition programme since it was rolled out as a complete package for eligible patients. That said, the investigators included all of the elements known to improve postdischarge outcomes in HF patients: patient education, medication and dietary monitoring, multidisciplinary teams providing HF case management and prompt follow-up with both specialists and primary care physicians after discharge. ${ }^{3} 1314$ Their ability to target their discharge intervention to those patients most likely to benefit is undoubtedly key to their impressive results. As even experienced clinicians are unable to accurately identify those patients at highest risk for readmission, ${ }^{15}$ efforts to duplicate the experience at Parkland in other settings must use tailored prediction models to identify those patients most at risk for adverse events post-discharge. While earlier models based solely on demographics and clinical factors demonstrated only fair ability to predict readmissions, ${ }^{16}$ Amarasingham and colleagues have demonstrated that 'second-generation' risk prediction models that also include social contextual factors and are generated in real time can be successfully used to target resources to improve post-discharge outcomes.

Whether the prognostic model used by Amarasingham and colleagues is generalisable to other HF patient cohorts (who are likely to be older and have different demographic/socioeconomic profiles) or applicable in other centres that do not collect some of the variables available in the Parkland Hospital electronic medical record (such as frequency of home address changes or number of missed clinic visits) is a question worthy of future study. Predicting the risk of post-discharge outcomes is an area of active research and a number of other 'second-generation' models to predict 30 -day readmission risk which take into account socioeconomic status and healthcare resource use before the index hospitalisation have been recently reported, with prediction characteristics similar to those for the model used by Amarasingham and colleagues. ${ }^{7}{ }^{17} 18$ Importantly, risk prediction efforts are increasingly focusing on identifying those patients with the highest modifiable risk for readmission. ${ }^{7}$

Amarasingham and colleagues have shown us that it is possible to identify those HF patients at highest risk for readmission prior to discharge and to deploy a multifaceted discharge transition programme to mitigate these risks. The challenge now is to follow their lead. However, the literature in this field has clearly shown us that one size does not fit all and implementation of readmission reduction strategies should be accompanied by robust evaluations of their impact.

Competing interests FAM holds career salary support from Alberta Innovates-Health Solutions and the Capital Health/ University of Alberta Chair in Cardiovascular Outcomes Research.
Provenance and peer review Not commissioned; internally peer reviewed.

\section{REFERENCES}

1 Jencks SF, Williams MV, Coleman EA. Rehospitalizations among patients in the medicare fee-for-service program. N Engl J Med 2009;360:1418-28.

2 Bueno H, Ross JS, Wang Y, et al. Trends in length of stay and short-term outcomes among Medicare patients hospitalized for heart failure, 1993-2006. JAMA 2010;303:2141-47.

3 Hansen LO, Young RS, Hinami K, et al. Interventions to reduce 30 day rehospitalization: a systematic review. Ann Intern Med 2011;155:520-8.

4 Amarasingham R, Patel P, Toto K, et al. Allocating scarce resources in real-time to reduce heart failure readmissions: a prospective, controlled study. BMJ Qual Saf 2013;22: 998-1005.

5 McAlister FA, Au A, Majumdar SR, et al. Teaching hospitals and weekday discharges are associated with better outcomes in heart failure. Circ Heart Fail 2013 Jun 28. [Epub ahead of print].

6 Van Walraven C, Bennett C, Jennings A, et al. Proportion of hospital readmissions deemed avoidable: a systematic review. CMAJ 2011;183:E391-402.

7 Donze J, Aujesky D, Williams D, et al. Potentially avoidable 30-day hospital readmissions in medical patients: derivation and validation of a prediction model. JAMA Intern Med 2013;173:632-8.

8 Joynt KE, Jha AK. Thirty day readmissions-truth and consequences. N Engl J Med 2012;366:1366-69.

9 Benbassat J, Taragin M. Hospital readmissions as a measure of quality of health care: advantages and limitations. Arch Intern Med 2000;160:1074-81.

10 Joynt KE, Jha AK. Who has higher readmission rates for heart failure, and why? Implications for efforts to improve care using financial incentives. Circ Cardiovasc Qual Outcomes 2011;4:53-9.

11 Kaboli PJ, Go JT, Hockenberry J, et al. Associations between reduced hospital length of stay and 30-day readmission rate and mortality: 14 year experience in 129 Veterans Affairs Hospitals. Ann Intern Med 2012;157:837-45.

12 Allen LA, Hernandez AF, Peterson ED, et al. Discharge to a skilled nursing facility and subsequent clinical outcomes among older patients hospitalized for heart failure/clinical perspective. Circ Heart Fail 2011;4:293-300.

13 Hernandez AF, Greiner MA, Fonarow Gc, et al. Relationship between early physician follow-up and 30-day readmission among medicare beneficiaries hospitalized for heart failure. JAMA 2010;303:1716-22.

14 McAlister FA, Stewart S, Ferrua S, et al. Multidisciplinary strategies for the management of heart failure patients at high risk for admission: a systematic review of randomized trials. J Am Coll Cardiol 2004;44:810-9.

15 Allaudeen N, Schnipper JL, Orav EJ, et al. Inability of providers to predict unplanned readmissions. J Gener Intern Med 2011;26:771-6.

16 Kansagara D, Englander H, Salanitro A, et al. Risk prediction models for hospital readmission. A systematic review. JAMA 2011;306:1688-98.

17 Eapen AJ, Liang L, Fonarow GC, et al. Validated, electronic health record deployable prediction models for assessing patient risk of 30-day rehospitalization and mortality in older heart failure patients. J Am Coll Cardiol HF 2013;1:245-51.

$18 \mathrm{Au}$ AG, McAlister FA, Bakal JA, et al. Predicting the risk of unplanned readmission or death within 30 days of discharge after a heart failure hospitalization. Am Heart J 2012;164:365-72. 